\title{
A Probabilistic Analysis Reveals Fundamental Limitations with the Environmental Impact Quotient and Similar Systems for Rating Pesticide Risks
}

Comparing risks among pesticides has substantial utility for decision makers. However, if rating schemes to compare risks are to be used, they must be conceptually and mathematically sound. We address limitations with pesticide risk rating schemes by examining in particular the Environmental Impact Quotient (EIQ) using, for the first time, a probabilistic analytic technique. To demonstrate the consequences of mapping discrete risk ratings to probabilities, adjusted EIQ's were calculated for a group of 20 insecticides in four chemical classes. Using Monte Carlo simulation, adjusted EIQ's were determined under different hypothetical scenarios by incorporating probability ranges. The analysis revealed that pesticides that have different EIQ's, and therefore different putative environmental effects, actually may be no different when incorporating uncertainty. The EIQ equation cannot take into account uncertainty the way that it is structured and provide reliable quotients of pesticide impact. The EIQ also is inconsistent with the accepted notion of risk as a joint probability of toxicity and exposure. Therefore, our results suggest that the EIQ and other similar schemes be discontinued in favor of conceptually sound schemes to estimate risk that rely on proper integration of toxicity and exposure information. 
1 A Probabilistic Analysis Reveals Fundamental Limitations with the Environmental Impact

2 Quotient and Similar Systems for Rating Pesticide Risks

3 Robert K. D. Peterson*, Jerome J. Schleier III

4 Department of Land Resources and Environmental Sciences, Montana State University,

5 Bozeman, Montana, USA 59717-3120

6 *Corresponding author, Robert K. D. Peterson. Department of Land Resources and

7 Environmental Sciences, Montana State University, Bozeman, Montana, 59717-3120, 406-994-

8 7927; bpeterson@montana.edu. 


\section{Introduction}

Numerous methods to rate pesticide risks have been introduced over the past two decades.

11 The methods are typically qualitative or semi-quantitative and involve rating and weighting

12 hazard, toxicity, and exposure factors for pesticide active ingredients. The purpose of these rating

13 schemes is to provide growers and other decision makers with information so that they can

14 discriminate among pesticides based on their risk to such entities as people, other non-target

15 organisms, and water quality.

16 Comparing risks among pesticides has substantial utility for decision makers (Peterson

17 2006). These comparisons are needed in addition to risk assessments of specific pesticides by

18 regulatory agencies. A regulatory agency, such as the U.S. Environmental Protection Agency,

19 should not be the sole arbiter of risk information and management decisions about pesticides.

20 However, if rating schemes to compare risks from pesticides are to be used, they must be

21 conceptually and mathematically sound.

The most influential scheme is arguably the Environmental Impact Quotient (EIQ) by

23 Kovach et al. (1992). Since the introduction of the EIQ, numerous researchers have evaluated it

24 or adapted it for their own risk rating schemes, or both (Cross \& Edwards-Jones 2011; Finizio et

25 al. 2001; Greitens \& Day 2007; Higley \& Wintersteen 1992; Labite et al. 2011; Leach \&

26 Mumford 2011; Maud et al. 2001; Muhammetoglu et al. 2010; Muhammetoglu \& Uslu 2007;

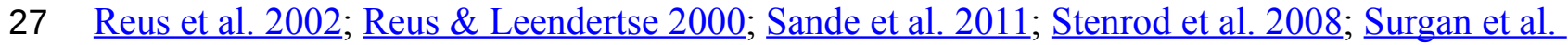

28 2010; van der Werf 1996; Vercruysse \& Steurbaut 2002; Yazgan \& Tanik 2005). In addition,

29 EIQ's for pesticides continue to be updated on a dedicated web site of the New York State

30 Integrated Pest Management Program, Cornell University

31 (www.nysipm.cornell.edu/publications/eiq/).

32 The EIQ method essentially is a mathematical formula that determines environmental

33 impact for pesticide active ingredients based on converting a raft of physicochemical and 
34 toxicological information, such as acute dermal toxicity, toxicity to birds, long-term health

35 effects, and soil runoff potential, into an arbitrary ratings scale of 1,3 , and 5 and then combining

36 and weighting those ratings through multiplication, division, and addition. This computation

37 results in EIQ's for farm worker, consumer, and environment. The EIQ's from these three

38 component categories are then averaged to determine a total EIQ. The EIQ equation is:

$\mathrm{EIQ}=([\mathrm{C}(\mathrm{DT} * 5+\mathrm{DT} * \mathrm{P})]+[(\mathrm{C} *((\mathrm{~S}+\mathrm{P} / 2) * \mathrm{SY})+(\mathrm{L})]+[(\mathrm{F} * \mathrm{R}+(\mathrm{D}((\mathrm{S}+\mathrm{P} / 2) * 3)+(\mathrm{Z} * \mathrm{P} * 3)+$

$(\mathrm{B} * \mathrm{P} * 5)]) / 3$

where: $\mathrm{C}=$ chronic toxicity, $\mathrm{DT}=$ dermal toxicity, $\mathrm{P}=$ plant surface half-life, $\mathrm{S}=$ soil

half-life, $\mathrm{SY}=$ systemicity, $\mathrm{L}=$ leaching potential, $\mathrm{F}=$ fish toxicity, $\mathrm{R}=$ surface loss potential, $\mathrm{D}$

43 = bird toxicity, $\mathrm{Z}=$ bee toxicity, $\mathrm{B}=$ beneficial arthropod toxicity.

Dushoff et al. (1994) critiqued the EIQ method, pointing out several conceptual problems

with the approach. Some shortcomings in the method were addressed in the original publication

(Kovach et al. 1992) and the problems discussed by Dushoff et al. (1994) were recognized by

47 Levitan et al. (1995). The critique by Dushoff et al. (1994) is compelling and suggests that the

48 EIQ method is substantially limited solely on the basis of conceptual problems with scaling and

49 weighting of the rating factors.

50 Cox et al. (2005) demonstrated mathematically that qualitative risk rating systems are

51 fundamentally limited because they do not adequately incorporate the key risk concept of

52 uncertainty. There are two major problems with qualitative risk rating systems: reversed rankings

53 and uninformative ratings. Reversed rankings occur when assigning a higher qualitative risk

54 rating to situations that have a lower quantitative risk. Uninformative ratings occur when

55 assigning the same qualitative ratings to risks that differ by many orders of magnitude. These

56 major limitations often obscure risk comparisons such that they are unable to distinguish between

57 risks. Moreover, Cox et al. (2005) argue that no consistent quantitative interpretation of

58 qualitative labels is possible and no change in how attributes are rated qualitatively can ensure 
59 that a qualitative rating system will give accurate results (but see Levine (2012) for a potential

60 solution using logarithmic scaling). Cox et al. (2005) argue that because of this, quantitative risk

61 models should be used instead of qualitative risk models. Since 2005, Cox and others have

62 expanded the analysis of risk rating systems (Barends et al. 2012; $\underline{\text { Cox 2008a; }}$ Cox 2008b; Cox

63 2009a; Cox 2009b; Levine 2012; Schleier III \& Peterson 2010; Schleier III et al. 2008).

64 Here, we examine pesticide risk rating schemes and the EIQ in particular using, for the

65 first time, a probabilistic analytic technique. Our purpose is not to repeat the mathematical proofs

66 of Coxr. et al. (2005) that clearly demonstrate, sensu lato, fundamental problems of qualitative

67 risk rating schemes. Rather, we will discuss how the problems extend to the EIQ using an

68 approach different from that taken by Dushoff et al. (1994). Furthermore, we discuss the

69 discontinuation of the EIQ and other similar schemes in favor of conceptually sound schemes to

70 estimate risk that rely on proper integration of toxicity and exposure information.

\section{Methods}

The ratings of 1, 3, and 5 in the EIQ method are surrogates for low, medium, and high risk

73 or impact or toxicity or persistence, depending on the factor of interest. For demonstration

74 purposes only, we show how converting the ratings to estimates of risk probabilities for only four

75 of the factors limits the value of the EIQ method. The EIQ factors, "long-term health effects,"

76 "leaching potential," and "surface runoff potential”, and ratings of "little-none," "possible,"

77 “definite," "small," "medium," and "large" imply that they are risks. Therefore, they have a

78 probability of occurrence rather than an absolute certainty of occurring. Similarly, the factor,

79 "beneficial arthropod toxicity" has ratings of "low impact," "moderate impact," and "severe

80 impact." Degrees of impact also have associated uncertainty.

81 Because the ratings of 1,3 , and 5 are surrogates for risk, they can be converted to risk

82 intervals that incorporate the underlying probabilities. Therefore, the simplest, yet coarse, way to

83 do this is to assume the ratings of 1,3 , and 5 span the range of risk from 0 to 1 (or 0 to $100 \%$ ). A 
84 rating of 1 , when mapped onto an interval of risks would be 0 to 0.32 . A score of 3 would be 0.33

85 to 0.66 and a score of 5 would be 0.67 to 1 . Consequently, if a pesticide has a "surface runoff

86 potential" factor that has a score of 3, it is at medium risk of runoff. However, a discrete score of

873 does not capture the probabilistic nature of risk, yet the score of 3 is intended to represent

88 medium risk. Therefore, the score needs to be mapped to an estimate of risk. This can be done

89 most simply by assuming a uniform probability density function of risk values from 0.32 to 0.66

90 for medium risk. Medium risk implies uncertainty and probability, but a score of 3 does not

91 accommodate that risk estimate. An interval of 0.33 to 0.66 , however crudely, accommodates the

92 probability of occurrence.

93 To demonstrate the consequences of mapping discrete risk ratings to probabilities, we

94 calculated adjusted EIQ's for a group of 20 actual insecticide active ingredients with unadjusted

95 EIQ's ranging from 22.1 (methiocarb) to 44 (diazinon). The insecticides evaluated were chosen

96 randomly from lists of active ingredients in $\mathrm{Yu}(\underline{2008})$, who provides a relatively complete list of

97 currently registered insecticides. Five insecticides each were chosen randomly from four

98 chemical classes: carbamates, neonicotinoids, organophosphates, and pyrethroids. The unadjusted

99 EIQ's and ratings were obtained from the New York State Integrated Pest Management Program,

100 Cornell University (www.nysipm.cornell.edu/publications/eiq/). The four factors discussed above

101 were converted to probability ranges of risk and all other factors were held constant at their

102 respective deterministic scores. To align those deterministic scores with the probability ranges

103 mapped for the four factors, the ratings were converted to static probabilities proportional to the

104 value of the scores. For example, a score of 3 for fish toxicity was converted to 0.5 .

105 Using Monte Carlo simulation (Oracle Crystal Ball ${ }^{\circledR} 11.2$, Denver, CO), we calculated

106 adjusted EIQ's under different hypothetical scenarios by incorporating the probability ranges

107 associated with the four factors (Fig. 1). Probabilities of occurrence of adjusted EIQ values were

108 determined by incorporating sampling from the statistical probability density function of each 
109

110

111 output reflected the probability of values that could occur.

\section{Results and Discussion}

113

114 (Fig. 1). For example, when incorporating uncertainty, adjusted EIQ's range from 0.75 to 1.17 for

115 cypermethrin and from 0.68 to 1.05 for acetamiprid. Therefore, more than $90 \%$ of the adjusted

116 EIQ values for these two insecticides overlap with each other. Yet, the unadjusted EIQ's are 36.4

117 and 28.7, respectively, a 7.7 EIQ unit difference.

118 Another example can be shown with imidacloprid and dinotefuran, two neonicotinoid

119 insecticides. The adjusted EIQ's range from 0.88 to 1.29 for imidacloprid and 0.65 to 1.04 for

120 dinotefuran. More than $26 \%$ of the adjusted EIQ values overlap with each other. The unadjusted

121 EIQ's are 36.7 and 22.3, respectively, a 14.4 EIQ unit difference. Consequently, these examples

122 show that pesticides with different EIQ's, and therefore different putative environmental effects,

123 actually may not be different because of the potential overlap in EIQ values when incorporating

124 uncertainty. Therefore, for example, a decision maker choosing acetamiprid over cypermethrin

125 because of the nearly 8-unit difference in EIQ's is choosing between two insecticides in which

126 there may be no difference in EIQ's when considering uncertainty (i.e., the EIQ's overlapped

$12790 \%$ of the time in the simulation).

128 Our results demonstrate the problems with qualitative risk ratings in which uncertainty is

129 not taken into account. Uncertainty cannot be ignored because the rating scores are surrogates for

130 probabilities of occurrence or impact. However, the EIQ equation cannot take into account

131 uncertainty the way that it is structured and provide reliable quotients of pesticide impact. As

132 demonstrated by Cox et al. (2005) in general, and by us in particular, the EIQ equation contains 
133 layers of qualitative coding which results in loss of information and inconsistency in the

134 interpretation of EIQ values.

135 In addition to the analyses above and those of Dushoff et al. (1994), the EIQ method is

136 limited because it does not properly incorporate exposure. Therefore, the EIQ is inconsistent with

137 the accepted notion of risk as a joint probability of toxicity and exposure. Because of this, the

138 method essentially is a hazard rating scheme, not a risk rating scheme. The method roughly

139 incorporates exposure by factoring scores for plant surface half-life, soil residue half-life,

140 leaching potential, and surface runoff potential into the equation, but these factors that certainly

141 influence exposure are proxies for exposure, not estimates of exposure. Similarly, the EIQ value

142 is adjusted to a field-use EIQ by incorporating application rate of the pesticide and percent active

143 ingredient in the formulation. This is particularly problematic because the adjustment to the EIQ

144 based on application rate has nothing to do with resulting risk, only the amount of environmental

145 loading of the pesticide. That is, a pesticide that is highly toxic at very low doses can have a low

146 use rate with a concomitant low field-use EIQ even though the exposure is sufficient to cause

147 unacceptable risks.

148 Cox et al. (2005), our findings presented here, and the conceptual problems pointed out by

149 Dushoff et al. (1994), preclude the use of the EIQ or other pesticide risk ratings that are

150 structured similarly to the EIQ. Dushoff et al. (1994) suggest various fixes, but many of these

151 suggestions commit the same mathematical errors as the original EIQ scheme. In addition,

152 different qualitative risk ranking systems can lead to different rankings of chemicals, and the

153 discrepancy in rankings cannot be resolved unless different qualitative risk ranking systems are

154 used together and evaluated, or a quantitative risk assessment is performed (Cox et al. 2005;

155 Morgan et al. 2000). The EIQ approach might have some utility for pesticides that are widely

156 separated in EIQ values, such as diazinon versus carbaryl, but the conceptual problems with the

157 scheme remain. 
If the EIQ method and others like it are not conceptually or mathematically sound, then

159 what should be used in their place? Risk is the joint probability of effect and exposure. In the case 160 of pesticides, risk is the joint probability of toxicity and exposure. Therefore, for risk rating

161 systems to be informative, toxicity and exposure must be integrated in an estimate of risk.

162 Risk rating systems for pesticides initially emerged when methods and models for

163 estimating environmental exposure were in nascent stages of development. However, the ability

164 to estimate the joint probability of exposure and toxicity (i.e., risk) currently is relatively simple

165 and there are several acceptable models for estimating environmental exposures, e.g., FOCUS,

166 PRZM-EXAMS, T-REX, (FOCUS 2001; USEPA 2005a; USEPA 2005b; USEPA 2005c; USEPA

167 2012).

168 The purpose of this article is not to examine a specific alternative to qualitative rating

169 systems for pesticides. However, a starting point to create a useful quantitative rating system is

170 the risk quotient (RQ) that is used in concept, but not necessarily by that specific term, by

171 regulatory agencies throughout the world. An RQ is simply the ratio of estimated or actual

172 environmental or dietary concentration of the pesticide to a toxic effect level or threshold. Some

173 other terms for this ratio include hazard quotient (HQ), hazard index (HI), margin of safety

174 (MOS), toxicity-exposure ratio (TER), and margin of exposure (MOE).

175 Peterson (2006) showed that an RQ approach is valuable for making direct comparisons

176 of quantitative risks between pesticides. Furthermore, Peterson (2006) demonstrated that a

177 numerical ranking of RQ's for the purpose of comparing risks is valid across different levels of

178 exposure refinement. Therefore, comparisons are equally valid whether using highly conservative

179 exposure estimates (i.e., tier 1) or actual environmental exposures (tier 4). However, higher tiers

180 should be used if the purpose is to accurately estimate the quantitative risk for an individual

181 pesticide within a specific use and location scenario. 
results suggest that qualitative rating systems should not be used for pesticide risk assessment,

management, or decision making because they cannot properly discriminate between different

185 levels of risk the way they are currently structured. We suggest that quantitative risk models be

186 used for both risk assessment and risk management of pesticides.

\section{Acknowledgements}

188 We thank L. G. Higley and S. H. Hutchins for their reviews of earlier versions of this 189 paper.

190 References

191 Barends DM, Oldenhof MT, Vredenbregt MJ, and Nauta MJ. 2012. Risk analysis of analytical validations by probabilistic modification of FMEA. Journal of Pharmaceutical and Biomedical Analysis 64-65:82-86.

Cox LA. 2008a. Some limitations of "Risk = Threat $\mathrm{x}$ Vulnerability x Consequence" for risk analysis of terrorist attacks. Risk Analysis 28:1749-1761.

Cox LA. 2008b. What's wrong with risk matrices? Risk Analysis 28:497-512.

197 Cox LA. 2009a. Some limitations of frequency as a component of risk: an expository note. Risk $198 \quad$ Analysis 29:171-175.

199 Cox LA. 2009b. What's wrong with hazard-ranking systems? An expository note. Risk Analysis 29:940-948.

201 Cox LA, Babayev D, and Huber W. 2005. Some limitations of qualitative risk rating systems. $202 \quad$ Risk Analysis 25:651-662.

203 Cross P, and Edwards-Jones G. 2011. Variation in pesticide hazard from arable crop production in 30:1579-1585. 
Dushoff J, Caldwell B, and Mohler CL. 1994. Evaluating the environmental effect of pesticides: a critique of the Environmental Impact Quotient. American Entomologist 40:180-184.

208

209

210

211

Finizio A, Calliera M, and Vighi M. 2001. Rating systems for pesticide risk classification on different ecosystems. Ecotoxicology and Environmental Safety 49:262-274.

FOCUS. 2001. FOCUS Surface Water Scenarios in the EU Evaluation Process under 91/414/EEC. Report of the FOCUS Working Group on Surface Water Scenarios. EC Document Reference SANCO/4802/2001-rev. 2.

Greitens TJ, and Day E. 2007. An alternative way to evaluate the environmental effects of integrated pest management: pesticide risk indicators. Renewable Agriculture and Food Systems 22:213-222.

Higley LG, and Wintersteen WK. 1992. A novel approach to environmental risk assessment of pesticides as a basis for incorporating environmental costs into econonomic injury levels. American Entomologist 38:34-39.

Kovach J, Petzoldt C, Degnil J, and Tette J. 1992. A method to measure the environmental impact of pesticides. New York's Food and Life Sciences Bulletin. Geneva, New York: Cornell University. p 8 .

Labite H, Butler F, and Cummins E. 2011. A review and evaluation of plant protection product ranking tools used in agriculture. Human and Ecological Risk Assessment 17:300-327.

Leach AW, and Mumford JD. 2011. Pesticide environmental accounting: a decision-making tool estimating external costs of pesticides. Journal Fur Verbraucherschutz Und Lebensmittelsicherheit-Journal of Consumer Protection and Food Safety 6:21-26.

Levine ES. 2012. Improving risk matrices: the advantages of logarithmically scaled axes. Journal of Risk Research 15:209-222. 
Levitan L, Merwin I, and Kovach J. 1995. Assessing the relative environmental impacts of agricultural pesticides: the quest for a holistic method. Agriculture, Ecosystems, and

232 233 Environment 55:153-168.

Maud J, Edwards-Jones G, and Quin F. 2001. Comparative evaluation of pesticide risk indices for policy development and assessment in the United Kingdom. Agriculture, Ecosystems and Environment 86:59-73.

Morgan MG, Florig HK, DeKay ML, and Fischbeck P. 2000. Categorizing risks for risk ranking. Risk Analysis 20:49-58.

Muhammetoglu A, Durmaz S, and Uslu B. 2010. Evaluation of the environmental impact of pesticides by application of three risk indicators. Environmental Forensics 11:179-186.

Muhammetoglu A, and Uslu B. 2007. Application of environmental impact quotient model to Kumluca region, Turkey to determine environmental impacts of pesticides. Water Science and Technology 56:139-145.

Peterson RKD. 2006. Comparing ecological risks of pesticides: the utility of a risk quotient ranking approach across refinements of exposure. Pest Management Science 62:46-56.

Reus J, Leendertse P, Bockstaller C, Fomsgaard I, Gutsche V, Lewis K, Nilsson C, Pussemier L, Trevisan M, van der Werf $\mathrm{H}$ et al. . 2002. Comparison and evaluation of eight pesticide environmental risk indicators developed in Europe and recommendations for future use. Agriculture, Ecosystems and Environment 90:177-187.

Reus JAWA, and Leendertse PC. 2000. The environmental yardstick for pesticides: a practical indicator used in The Netherlands. Crop Protection 19:637-641.

Sande D, Mullen J, Wetzstein M, and Houston J. 2011. Environmental impacts from pesticide use: a case study of soil fumigation in Florida tomato production. International Journal of Environmental Research and Public Health 8:4649-4661. 
253 Schleier III JJ, and Peterson RKD. 2010. Limitations of the Entomological Operational Risk

254

255

256

257

258

259

260

261

262

263

264

265

266

267

268

269

270

271

272

273

274

275

276

277

Assessment using probabilistic and deterministic analyses. Military Medicine 175:594598.

Schleier III JJ, Sing SE, and Peterson RKD. 2008. Regional ecological risk assessment for the introduction of Gambusia affinis (western mosquitofish) into Montana watersheds. Biological Invasions 10:1277-1287.

Stenrod M, Heggen HE, Bolli RI, and Eklo OM. 2008. Testing and comparison of three pesticide risk indicator models under Norwegian conditions - A case study in the Skuterud and Heiabekken catchments. Agriculture Ecosystems \& Environment 123:15-29.

Surgan M, Condon M, and Cox C. 2010. Pesticide risk indicators: unidentified inert ingredients compromise their integrity and utility. Environmental Management 45:834-841.

USEPA. 2005a. The Exposure Analysis Modeling System v. 2.98.04.06. Available at http://www.epa.gov/ceampubl/swater/express/index.htm (accessed 31 August 2005).

USEPA. 2005b. EXPRESS v. 1.00.00.12. Available at http://www.epa.gov/ceampubl/swater/express/index.htm (accessed 31 August 2005).

USEPA. 2005c. Pesticide Root Zone Model v. 3.12.3. Available at http://www.epa.gov/ceampubl/swater/express/index.htm (accessed 31 August 2005).

USEPA. 2012. T-REX (Terrestrial Residue EXposure Model). Available at http://www.epa.gov/oppefed 1/models/terrestrial/trex/t rex user guide.htm.

van der Werf HMG. 1996. Assessing the impact of pesticides on the environment. Agriculture, Ecosystems and Environment 60:81-96.

Vercruysse F, and Steurbaut W. 2002. POCER, the pesticide occupational and environmental risk indicator. Crop Protection 21:307-315.

Yazgan MS, and Tanik A. 2005. A new approach for calculating the relative risk level of pesticides. Environment International 31:687-692. 
278 Yu SJ. 2008. The toxicology and biochemistry of insecticides. Boca Raton, FL, USA: CRC Press. 


\section{Figure Caption}

280 Figure 1: Adjusted Environmental Impact Quotient (EIQ) values for 20 insecticides based

281 on probabilistic simulation analysis. For each bar, the bottom line is the $10^{\text {th }}$, the middle line is

282 the $50^{\text {th }}$, and the top line is the $90^{\text {th }}$ percentile value from the simulation. The number at the top of

283 each bar is the original EIQ value. The original EIQ value reported for naled, 49, is incorrect. The

284 correct value is 41 . 


\section{Figure 1}

Adjusted Environmental Impact Quotient (EIQ) values for 20 insecticides based on probabilistic simulation analysis

For each bar, the bottom line is the $10^{\text {th }}$, the middle line is the $50^{\text {th }}$, and the top line is the $90^{\text {th }}$ percentile value from the simulation. The number at the top of each bar is the original EIQ value. The original EIQ value reported for naled, 49, is incorrect. The correct value is 41 .

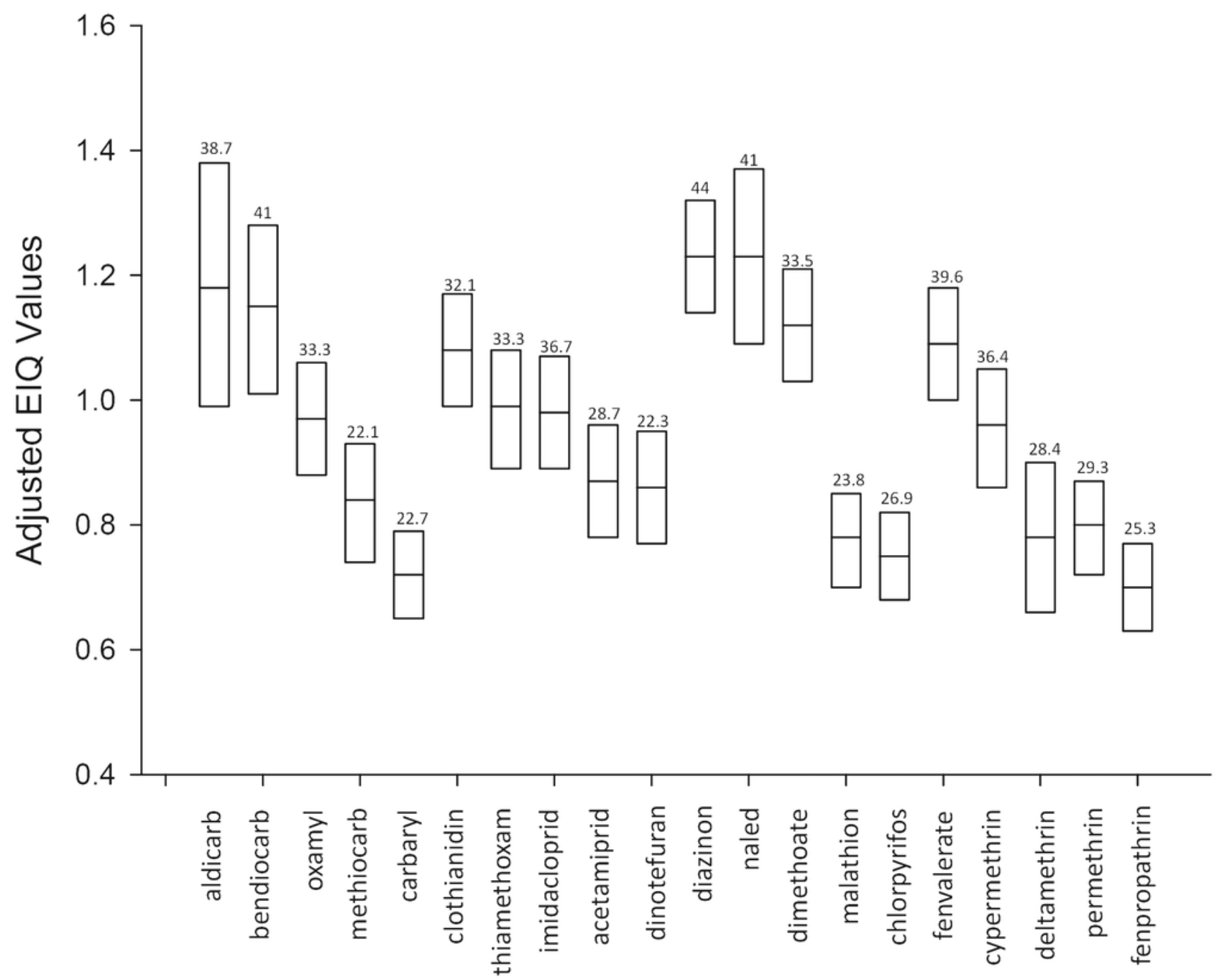

